\title{
Habilidades metodológicas para la comunicación científica y competencias laborales en docentes universitarios
}

\author{
Scientific communication in favor of methodological skills labor \\ competences in university teachers \\ Recibido: febrero 02 de 2020 | Revisado: febrero 27 de 2020 | Aceptado: marzo 09 de 2020
}

\author{
George Argota PéreZ \\ Rosalvina Campos Pérez \\ Ramiro M. Yallico CaLmetT ${ }^{3}$ \\ María H. Quispe RevatTa \\ Marco R. Revatta Salas ${ }^{3}$ \\ Luis Celi SaAvedra ${ }^{4}$
}

1 Centro de Investigaciones Avanzadas y Formación Superior en Educación, Salud y Medio Ambiente "AMTAWI". Puno, Perú george.argota@gmail.com

2 Universidad Nacional Federico Villarreal

Lima, Perú, Facultad de Humanidades rcampos@unfv.edu.pe

3 Universidad Nacional "San Luis Gonzaga” (UNICA). Ica, Perú Facultad de Medicina Humana

maria.quispe@unica.edu.pe marco.revatta@unica.edu.pe Facultad de Ciencia de la Educación y Humanidades ereyece@hotmail.com

4 Universidad de San Martín de Porres, Lima, Perú Facultad de Ingeniería y Arquitectura lcelis@usmp.pe

\begin{abstract}
Resumen
La búsqueda de estrategias que permitan reconocer la destreza profesional con base en el dominio de las estructuras metodológicas y que se expresen formas escritas continúa siendo un desafío. El objetivo del estudio fue evaluar las habilidades metodológicas para la comunicación científica y competencias laborales. En febrero de 2020, se seleccionó mediante un muestreo probabilístico aleatorio ocho docentes universitarios en formación posgraduada de la Universidad Nacional "San Luis Gonzaga", Ica-Perú. Se analizó las habilidades metodológicas, además, de la comunicación científica y competencias laborales mediante un análisis de descripción según las respuestas a preguntas indicadas. Se planteó y aceptó como hipótesis nula que, las habilidades metodológicas para la comunicación científica y competencias laborales de docentes universitarios en formación posgraduada son limitadas $\left(\mathrm{H}_{0}: \mu=16 ; \mathrm{H}_{1}\right.$ : $\mu>16$ ) obteniéndose un promedio de 13,875 puntos. Se indicó de forma inmediata, establecer estrategias de enseñanza que permitan contribuir al aprendizaje básico de la metodología dela investigación y sus modalidades de comunicación escrita de cualquier resultado para el reconocimiento de las competencias laborales. Se concluyó que, las habilidades metodológicas para la comunicación científica y competencias laborales de los docentes universitarios en formación posgraduada fueron reducidas.
\end{abstract}

Palabras clave: competencias, enseñanza pedagógica, estrategias, habilidades, redacción

\section{Abstract}

The search for strategies that allow to recognize professional skill based on the mastery of methodological structures and that written forms are expressed continues to be a challenge. In this paper was evaluate methodological skills for scientific communication and job skills. In February 2020, eight-university faculty in post-graduate training from the National University of San Luis Gonzaga, Ica-Peru, was selected by random probabilistic sampling. Methodological skills were also analyzed in addition to scientific communication and work skills by means of a description analysis according to the answers to the indicated questions. Methodological skills for scientific communication and job skills of university teachers in post- graduate training were raised and accepted as a null hypothesis are limited $\left(\mathrm{H}_{0}: \mu=16 ; \mathrm{H}_{1}: \mu>16\right)$ earning an average of 13,875 points. It was immediately indicated to establish teaching strategies to contribute to the basic learning of the research methodology and its modalities of written communication of any outcome for

(C) Los autores. Este artículo es publicado por la Revista Campus de la Facultad de Ingeniería y Arquitectura de la Universidad de San Martín de Porres. Este artículo se distribuye en los términos de la Licencia Creative Commons Atribución No-comercial - Compartir-Igual 4.0 Internacional (https://creativecommons.org/licenses/ CC-BY), que permite el uso no comercial, distribución y reproducción en cualquier medio siempre que la obra original sea debidamente citada. Para uso comercial contactar a: revistacampus@usmp.pe. 
the recognition of labor competences. It was concluded that the methodological skills for scientific communication and job skills of university teachers in post-graduate training were reduced.

Key words: competencies, pedagogical teaching, strategies, skills, writing

\section{Introducción}

Más de 2.5 millones de artículos científicos se publica cada año (Ware \& Mabe, 2015) siendo la consistencia del contenido (Onodera \& Yoshikane, 2015; Van Steendam, 2016) y coherencia de la redacción, el principal mérito de los manuscritos (Bremner et al., 2014; Auvinen, 2015; Amjad et al., 2017). La búsqueda e interpretación exhaustiva de la literatura científica posibilita un nuevo conocimiento dialogado (Hori \& Hori, 2017) y posteriormente, otra publicación se favorece de forma construida (Belcher, Barron \& Yang, 2016) siendo valioso, la probabilidad de volver asumirse la pretensión como calidad final diferenciada (Lin \& Evans, 2012; Lillis \& Curry, 2015).

En ese escenario, la expresión de valoraciones sociales puede analizarse a partir, de las múltiples investigaciones universitarias que se producen (Too \& Bajracharya, 2015; Adams et al., 2018) constituyen un aval de decisiones (Disterheft et al., 2016), además, de la participación como grupos de interés contribuyentes al cambio por parte de los estudiantes (Turan et al., 2016). La expresión directa que genera impacto social está dada por la participación y orientación de los docentes universitarios (Cotton et al., 2016; Ting \& Cheng, 2017) donde cualquier reto para su empleo laboral en el presente y probables futuros dependen de las habilidades. Es importante mencionar que el empleo laboral requiere habilidades de comunicación, desempeño en equipo y destreza tecnológica (McMurray et al., 2016).

De igual modo, el aprendizaje cognitivo que se adquiere debe ser acompañado por la propia autorrealización y el desarrollo de habilidades prácticas (Scott, 2017) que contribuyan a la resolución de problemas mediante el razonamiento lógico transferibles (Weinstein,2012). Así mismo, desarrollar habilidades basado en el pensamiento crítico (Canziani \& Tullar, 2017) exige la búsqueda de información (Ungaretti et al., 2015), reconocimiento del aprendizaje continuo y su regulación. La formación y conceptualización de todo hallazgo durante el proceso de adquisición de habilidades tiene su base en el procedimiento interactivo (Teckchandani \& Khanin, 2014) donde cualquier percepción puede definirse y corregirse (Chang \& Rieple, 2013).

El objetivo de nuestra investigación fue evaluar las habilidades metodológicas para la comunicación científica y competencias laborales.

\section{Método}

El estudio se realizó en febrero de 2020 con docentes universitarios en formación posgraduada de la Universidad Nacional "San Luis Gonzaga“ de Ica, Perú. Se seleccionó una muestra probabilística aleatoria de ocho docentes donde se analizó, las habilidades metodológicas relacionadas con dos evaluaciones (Tabla 1). 
Tabla 1

Habilidades metodológicas / docentes universitarios

1. Si usted realiza algún tipo de estudio o investigación mencione:

a) ¿Cuál es el problema científico que aborda y que aspectos le indicaron para ello?

b) ¿Cuál es la suposición o conjetura de solución al problema planteado?

c) ¿Qué objetivos expresa cumplimentar?

d) ¿Qué tipo de investigación consideras realizar?

e) ¿Qué métodos y técnicas describes o empleas para su realización?

2. Describa las siguientes condiciones:

a) Cualidad de la proposición

b) Objetivo de la lógica y aplicación en su área de investigación

Para reconocer la comunicación científica y competencias laborales se analizó el vínculo a investigaciones y ejecución de proyectos (Tabla 2).

Tabla 2

Comunicación cientifica y competencias laborales / docentes universitarios

1. Marque con una X, cuáles labores realiza en la actualidad.

- Proyecto de tesis (programa de maestría)

- Proyecto de tesis (programa de doctorado)

- Investigación básica

- Investigación aplicada

- Acciones de proyectos (investigador principal)

- Acciones de proyectos (investigador participante) _-

- No vinculado

Se planteó como hipótesis: Ho: Las habilidades metodológicas para la comunicación científica y competencias laborales de docentes universitarios en formación posgraduada son limitadas. H1:Lashabilidades metodológicas parala comunicación científica y competencias laborales de docentes universitarios en formación posgraduada no son limitadas. Adicionalmente, se utilizó el programa estadístico profesional Epidat 4.2 (versión libre) donde la prueba de normalidad fue Kolmogorov-Smirnov. Se realizó un análisis de distribución $t$, además, de un análisis t para 1 muestra con nueve grados de libertad y prueba de hipótesis con criterio de 90 puntos (media hipotética) considerando significativos los resultados cuando $\mathrm{p}<$ 0.05 .

\section{Resultados}

La Tabla 3 muestra la puntuación de los docentes universitarios en formación posgraduada con relación a la evaluación metodológica de la comunicación científica y competencias laborales. 
George Argota Pérez - Rosalvina Campos Pérez - Ramiro M. Yallico Calmett -

María H. Quispe Revatta - Marco R. Revatta Salas - Luis Celi Saavedra

Tabla 3

Puntuación promedio / estudiantes universitarios de formación posgraduada $=$ EUFP / evaluación metodológica

\begin{tabular}{ccccccc}
\hline EUFP & $1 \mathrm{a}$ & $1 \mathrm{~b}$ & $1 \mathrm{c}$ & $1 \mathrm{~d}$ & $1 \mathrm{e}$ & total \\
\hline 1 & 15 & 14 & 15 & 14 & 14 & 72 \\
2 & 13 & 11 & 13 & 13 & 13 & 63 \\
3 & 14 & 13 & 13 & 14 & 14 & 68 \\
4 & 15 & 15 & 15 & 15 & 13 & 73 \\
5 & 14 & 14 & 14 & 14 & 14 & 70 \\
6 & 15 & 15 & 14 & 14 & 13 & 71 \\
7 & 14 & 14 & 14 & 14 & 14 & 70 \\
8 & 13 & 14 & 13 & 15 & 13 & 68 \\
Total & 14,125 & 13,75 & 13,875 & 14,125 & 13,5 & 69,375 \\
Desviación Estándar & 0,834 & 1,281 & 0,834 & 0,640 & 0,534 & 3,113 \\
\hline
\end{tabular}

La Figura 1 muestra la distribución comunicación científica y competencias t según el total de puntuación otorgada para la evaluación metodológica de la formación posgraduada.

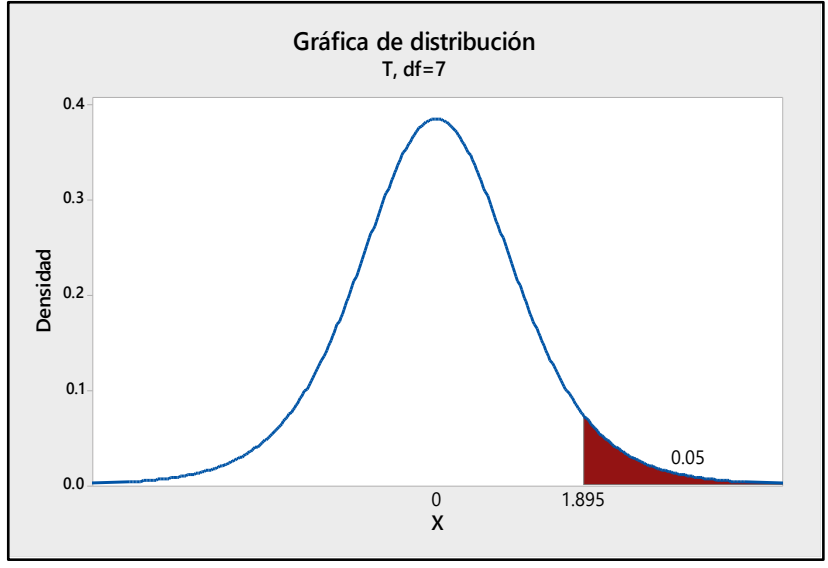

Figura 1. Distribución t / puntuación otorgada.

La Tabla 4 muestra el análisis de la estadística descriptiva según la puntuación promedio de los estudiantes universitarios de formación posgraduada

Tabla 4

Análisis de estadística descriptiva

\begin{tabular}{l|c|c|c|c|c|c}
\hline Variable & $\mathrm{N}$ & $\begin{array}{c}\text { Media de } \\
\text { puntuación }\end{array}$ & $\begin{array}{c}\text { Error de la } \\
\text { media }\end{array}$ & $\begin{array}{c}\text { Desviación } \\
\text { estándar }\end{array}$ & $\begin{array}{c}\text { Coeficiente de } \\
\text { variación }\end{array}$ & Asimetría \\
\hline Investigadores & 8 & 13,875 & 1,100 & 3,113 & 4,488 & $-1,222$ \\
\hline & \multicolumn{2}{c}{$\mathrm{H}_{0}: \mu=16$} & Valor T & \multicolumn{2}{c}{ Valor $\mathrm{p}$} \\
Hipótesis nula & $\mathrm{H}_{1}: \mu>16$ & 48,48 & \multicolumn{2}{c}{0.000} \\
Hipótesis alterna
\end{tabular}




\section{Discusión}

Se observó, puntuación baja de los docentes universitarios en formación posgraduada con relación a la evaluación metodológica de la comunicación científica y competencias laborales donde se esperó, promedio de puntuación mayor a 16 por cuanto, la hipótesis $\mathrm{H} 1$ se aceptó. Por lo general, existe la tendencia en los docentes universitarios a considerarse investigadores, pero como indica (McDonnell, 2017), interesarse en la carrera científicoacadémica y por ende, redactor de manuscritos científicos, es concebir que la escritura, significa un desafío en el desarrollo humano pero resulta imposible comunicar cualquier resultado, si se desconoce la estructura de investigación. En tal sentido, dos interrogantes pueden mencionarse según los resultados hallados:

1. ¿Qué habilidades metodológicas presentan los docentes universitarios para la comunicación científica?

2. ¿Cuáles son las competencias laborales en docentes universitarios basadas en la comunicación científica?

Las respuestas pueden ser diversas, pero una de las razones comunes entre las habilidades metodológicas y las competencias laborales considerándose la comunicación científica está en lo esencial que es, práctica coincidiendo con Brunhaver et al., (2017).

Mohan et al., (2010) indican que, la mayoría de las habilidades aprendidas en la educación superior universitaria son analíticas y de resolución de problemas, pero podrían resultar ineficaces si no existe el acompańamiento necesario de las habilidades profesionales. Por lo general, son indicativos las posibles habilidades metodológicasquelosdocentesuniversitarios adquieren durante un aprendizaje pasivo como un curso donde se evidencia la elevada capacidad técnica, pero, plasmar en múltiples ocasiones lo que se expresa como habilidades de comunicación en la escritura, sigue siendo limitado. Fletcher et al., (2017) en su estudio direccionado a las habilidades de empleabilidad destacaron la necesidad de desarrollar capacitaciones en habilidades transferibles de modo que, fue observado en este estudio la no correspondencia entre el saber conocer y el saber hacer.

Azizan et al., (2018) señalan que, la investigación contemporánea debe concentrarse en metodologías pedagógicas que resulten innovadoras para garantizar el interés del aprendizaje cooperativo, el aprendizaje basado en problemas, la cooperación interdisciplinaria entre otros pero debe mencionarse lo inmediato en establecer estrategias de enseñanza que permitan contribuir al aprendizaje básico de la metodología de la investigación y sus modalidades de comunicar cualquier resultado para el reconocimiento de las competencias laborales.

Uno de las principales limitaciones en el estudio fue registrar y describir la experiencia profesional relacionada con la publicación de artículos científicos en revistas indexadas y que permitieran hacer un análisis exhaustivo para valorar por clases el dominio de otras estructuras metodológicas con creatividades en la comunicación científica y su reconocimiento social.

\section{Conclusiones}

Se concluye que, las habilidades metodológicas para la comunicación científica y competencias laborales de los docentes universitarios en formación posgraduada fueron reducidas. 


\section{Referencias}

Adams, R.; Martin, S. \& Boom, K. (2018). University culture and sustainability: Designing and implementing an enabling framework. Journal of Cleaner Production; 171, 434- 445. Doi. org/10.1016/j.jclepro.2017.10.032

Amjad, T., Ding, Y., Xu, J., Zhang, C., Daud, A., Tang, J. \& Song, M. (2017). Standing on the shoulders of giants. Journal of Informetrics; 11(1), 307-323. Doi: 10.1016/j. joi.2017.01.004

Auvinen, A. (2015). How do I write a scientific article-advice to a young researcher. Duodecim; 131(16), 1460-1466. Disponible en: https://www.ncbi.nlm.nih.gov/ pubmed/26485939

Azizan, M.T., Mellon, N., Ramli, R.M. \& Yusup, S. (2018). Improving teamwork skills and enhancing deep learning via development of board game using cooperative learning method in Reaction Engineering course. Educ. Chem. Eng; 22, 1-13. Doi.org/10.1016/J. ECE.2017.10.002

Belcher, D.D., Barron, S.F.H. \& Yang, H.S. (2016). English for professional academic purposes. In: Shaw, P., Hyland, K. (Eds.). The Routledge handbook of English for Academic Purposes. Routledge, Abingdon, Oxon; 502-514.

Bremner, S., Pierson, S.A., Jones, R. \& Bhatia, V. (2014). Task design and interaction in collaborative writing:
The students' story. Bus. Profess. Commun. Quart, 77(2), 150- 168. Doi: $10.1177 / 2329490613514598$

Brunhaver, S.R., Korte, R.F., Barley, S.R. \& Sheppard, S.D. (2017). Bridging the gaps between engineering education and practice. In: U.S. Engineering in a Global Economy. University of Chicago Press, 129-163.

Canziani, B. \& Tullar, W.L. (2017). Developing Critical Thinking through Student Consulting Projects. Journal of Education for Business; 92(6), 271-279. Doi: $.1080 / 08832323.2017 .1345849$

Cotton, D.; Shiel, C. \& Paco, A. (2016). Energy saving on campus: a comparison of students'attitudes and reported behaviours in th UK and Portugal. Journal of Cleaner Production; 129, 586-595. Doi: 10.1016/j.jclepro.2016.03.136

Disterheft, A.; Caeiro, S.; Leal, F.W. \& Azeiteiro, U.M. (2016). The INDICARE-model - measuring and caring about participation in higher education's sustainability assessment. Ecological Indicators; 63, 172-186. Doi: 10.1016/j. ecolind.2015.11.057

Fletcher, A.J., Sharif, A.W.A. \& Haw, M.D. (2017). Using the perceptions of chemical engineering students and graduates to develop employability skills. Educ. Chem. Eng; 18, 11-25. Doi: 10.1016/J. ECE.2016.07.001

Hori, C. \& Hori, T. (2017). End-to-end conversation modeling track in 
dstc6. 1-8. Disponible en: http:// workshop.colips.org/dstc6/papers/ track2_overview_hori.pdf

Lillis, T. \& Curry, M.J. (2015). The politics of English, language and uptake. The case of international academic journal article reviews. The politics of English, language and uptake; 28, 127-150. Doi: 10.1075/aila.28.06lil

Lin, L. \& Evans, S. (2012). Structural patterns in empirical research articles: A crossdisciplinary study. English for Specific Purposes; 31, 150-160. Doi: 10.1016/j. esp.2011.10.002

McMurray, S., Dutton, M., McQuaid, R. \& Richard, A. (2016). Employer demands from business graduates. Education + Training; 58(1), 112132. Disponible en. https://eric. ed.gov/?id=EJ1086016

Mohan, A., Merle, D., Jackson, C., Lannin, J. \& Nair, S.S. (2010). Professional skills in the engineering curriculum. IEEE Trans. Educ; 53, 562-571, Doi: 10.1109/ TE.2009.2033041

Onodera, N. \& Yoshikane, F. (2015). Factors affecting citation rates of research articles. Journal of the Association for Information Science and Technology; 66(4), 739-764.

Doi: 10.1002/asi.23209

Scott, K. S. (2017). An integrative framework for problembased learning and action learning: Promoting evidencebased design and evaluation in leadership development. Human Resource Development Review; 16(1), 3-34. Doi: $0.1177 \%$ 2F1534484317693090

Teckchandani, A., \& Khanin, D. (2014). The instructor's role in the student consulting process: Working with the student team. Small Business Institute Journal; 10(1), 11-24. Doi: 10.5465/ AMBPP.2014.13187abstract

Ting, D.H. \& Cheng, C.F.C. (2017). Measuring the marginal effect of pro-environmental behaviour: Guided learning and behavioural enhancement. Journal of Hospitality Leisure, Sport \& Tourism Education; 20, 16-26. Doi: 10.1016/j. jhlste.2016.12.001

Too, L. \& Bajracharya, B. (2015). Sustainable campus: engaging the community in sustainability. International Journal of Sustainability in Higher Education; 16, 57-71. Doi: 10.1108/ IJSHE-07-2013-0080

Turan, F.K.; Cetinkaya, S. \& Ustun, C. (2016). A methodological framework to analyze stakeholder preferences and propose strategic pathways for a sustainable university. Higher Education; 72,743-760. Doi: 10.1007/ s10734-015-9973-8

Ungaretti, T., Thompson, K. R., Miller, A. \& Peterson, T. O. (2015). Problembased learning: Lessons from medical education and challenges for management education. Academy of Management Learning 
and Education; 14(2), 173-186. Doi: $10.5465 /$ amle.2013.0245

Van Steendam, E. (2016). Editorial: forms of collaboration in writing. J. Writing Res; 8(2), 183-204. Doi: 10.17239 / jowr-2016.08.02.01

Ware, M. \& Mabe, M. (2015). The STM Report an Overview of Scientific and Scholarly Journal Publishing. (The Hague, Netherlands). University of Nebraska-Lincoln,USA.Recuperado en: https://digitalcommons. unl.edu/cgi/viewcontent. cgi ?article $=1008 \&$ context =scholcom Weinstein, K. (2012). Action learning: A practical guide. Aldershot: Gower Publishing.

Yeadon-Lee, A. (2013). Action learning: The possibility of differing hierarchies in learning sets. Action Learning: Research and Practice; 10(1), 39-53. Disponible en: https://eric.ed.gov/?id=EJ995342 